\title{
Artificial Neural Network based COVID-19 Suspected Area Identification
}

\author{
Tanjima Akhter ${ }^{1}$, Md. Ariful Islam², Saiful Islam ${ }^{3}$ \\ ${ }^{1}$ Department of Applied Mathematics, Noakhali Science \& Technology University, Bangladesh \\ ${ }^{2}$ Department of Robotics \& Mechatronics Engineering, University of Dhaka, Bangladesh \\ ${ }^{3}$ Department of Information and Communication Technology, Bangladesh University of Professionals, Bangladesh
}

Received: November 24, 2020, Revised: December 18, 2020, Accepted: December 19, 2020, Available Online: December 28,2020

\begin{abstract}
This paper deals with the symptoms based COVID-19 suspected area identification using an artificial neural network by which a country or region can be divided into red, yellow, and green zone representing the highly infected area, moderate infected area, and controlled or low infected area, respectively. At first, an online survey of twenty (20) patients was conducted based on the nine (09) major symptoms of COVID-19. Then, a model based on the fuzzy logic system was designed consisting of COVID-19 symptoms identification, fuzzification, rule evaluation, fuzzy inference mechanism, etc. for getting the data sets to be trained in neural networks. For different combinations of 09 symptoms, different rules were generated and evaluated for possible recommendations. Based on different rules, three possible outputs representing high infection probability, medium infection probability, and low infection probability were obtained using the Mamdani inference mechanism. These outputs were termed as red, yellow, and green zone separated by the crisp value of $+1,0,-1$, respectively, and considered as target data to be trained in neural networks.
\end{abstract}

Keywords: Neural Network; COVID-19; Fuzzy Logic System; Mamdani Inference Mechanism.

This work is licensed under a Creative Commons Attribution-Non Commercial 4.0 International

\section{Introduction}

In Wuhan, China a novel corona virus named COVID-19 was discovered in early December 2019 and since then it has been spreading out rapidly throughout the world. Several viruses have appeared in the history of humanity since the beginning of civilization, COVID-19 is one of few which has been declared as 'Pandemic' throughout the world by WHO because of its infection nature. Nowadays, it becomes a major health concern which is causing several health issues in human beings. Till October 29, 2020, the number of confirmed cases is 44 million while the death toll reaches 1.17 million and the number of recovered cases is 29.8 million around the world [1]. COVID-19 has affected 216 countries and territories around the world [1]. Among all 216 countries USA, India, Brazil, Italy, Spain, Russia, the UK have been identified as the hotspot with the most confirmed cases and the infections are increasing exponentially. This is an alarming situation for the whole world and moreover, the situation is getting out of control for almost every country whether it's a rich or developing/poor one. It's affecting overall infrastructure, economic, social and other aspects of a country as to reduce the infection rate most of the country were compelled to declare a state of emergency and have locked down cities.

COVID-19 is a zoonotic type which can transmit the infection from animal to human but now infection is spreading from human to human and each infected human can infect more than two. In this way further infections may occur, and the infection rate has been identified as an exponential one [2]. Researchers and Scientists around the world were unable to find any specific treatment for COVID-19 so far but they are working hard and soul to find the vaccine for the prevention of this disease [2]. Several shreds of evidences predicted, Wuhan City, as the source of this outbreak and it has been identified as one of the significant role-playing places in the initial amplification of the outbreak.
The first identified case of COVID-19 subsequently named as SARS-COV-2 was reported by the official of Wuhan city, China in December 2019 [3].

Although COVID-19 causes mild illness for people with strong immunity, it may make some people very ill especially older people and people with pre-existing medical conditions appear to be more vulnerable and the disease can be fatal for them [4]. Fever, trouble breathing, chest pain, dry cough, diarrhea, sore throat, and headaches are known to be common symptoms of COVID-19. However, in some cases, it can cause pneumonia and kidney failure even no symptoms at all. People are dying every single day upon getting the infection. According to the study by Centers for Disease Control and Prevention (CDC) between two days to two weeks of exposure to the virus symptoms usually appear [5].

In processing huge data by analyzing computationally to reveal projection, prediction, and patterns, Artificial Neural Network has proven to be one of the efficient and timeconsuming ones. It has a greater and enriching prospect not only in the engineering sector but also in the medical sector [6]. This study provides neural network-based logical decisions based on COVID-19 symptoms by which a country or region can be divided into red, yellow, and green zone representing highly infected, moderate infected, and controlled or low infected areas respectively. An algorithm based on neural network has proposed to give overall statistics of a country about low, medium, and high density of infected people. Three networks have generated to compare the proposed model and the Feed forward back propagation algorithm has implemented in these networks. The fuzzy logic output has trained into the neural network for which the threshold value of separating different places of a country can be categorized into three zones. MATLAB has used for testing algorithm and obtaining outcomes. 


\section{Methodology}

In this paper, we propose a model using the artificial neural network by which the identification of COVID-19 suspected area based on COVID-19 symptoms can be realized easily. The flow chart of this model is shown in Fig. 1.

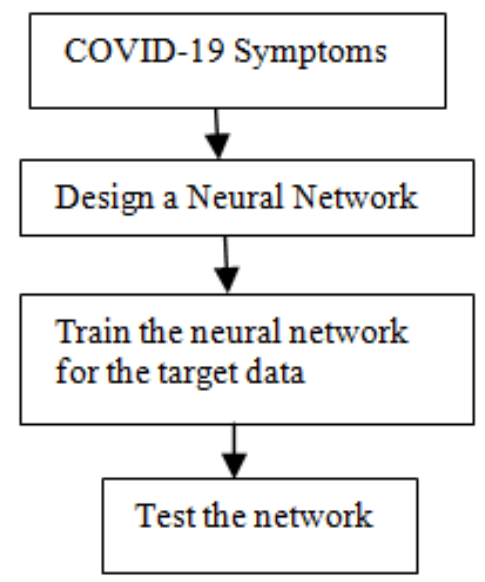

Fig. 1 Proposed algorithm for identification of danger zones of COVID-19

\subsection{COVID-19 Symptoms}

To train the neural network, we have generated data sets from the different combinations of COVID-19 symptoms. The major symptoms of COVID-19 are fever or body temperature (BT), dry cough (DC), trouble breathing (TB), chest pain (CP), diarrhea, sore throat (ST), and headache, etc. [7], [8]. If a person gets close contact to the infected person having these symptoms or travel to the infected area may become COVID-19 positive or negative. Based on these causes and symptoms of COVID-19, at first, an online survey of twenty (20) patients was conducted, recorded, and analysed. In most of the cases, patients having a severe level of breathing problems, chest pain, dry cough, and get close contact with the infected person were identified as COVID-19 positive while a few of them were resulted as COVID-19 negative despite having headache, fever, or sore throat. This survey information was implemented in the Mamdani inference system as shown in Fig. 2. Here, the symptoms level of COVID-19 was considered as linguistic variables and their corresponding possible output recommendations were recorded. Then, different fuzzy rules were inserted in the Mamdani inference system and evaluated for the possible recommendations depicted in Fig. 3.

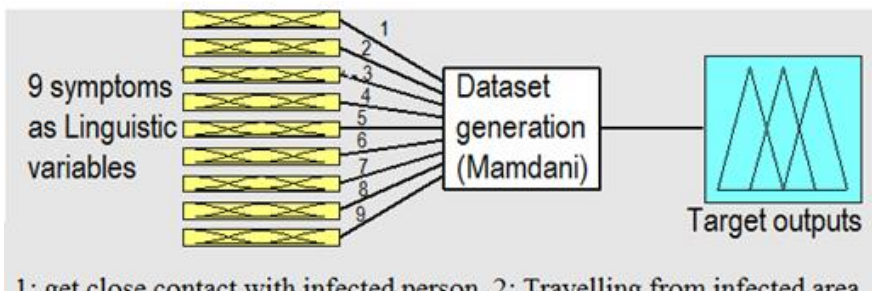

1: get close contact with infected person, 2 : Travelling from infected area recently, 3 : fever or temperature of the body, 4 : chest pain, 5: diarrhea, 6: dry cough, 7: trouble breathing, 8: sore throat, 9 : headache

Fig. 2 Mamdani inference system for creating COVID-19 symptoms data set

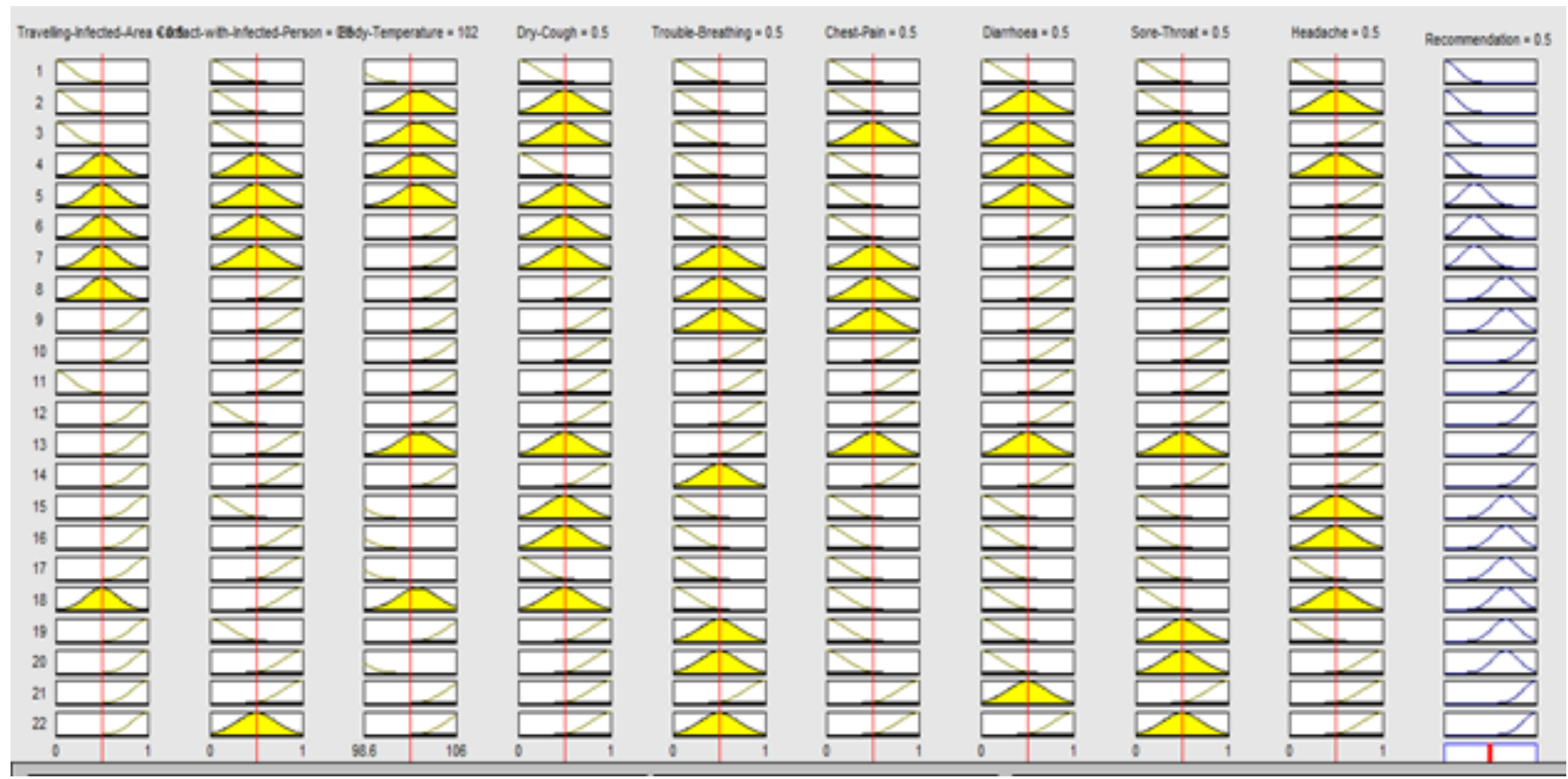

Fig. 3 Rule evaluation for possible recommendation 


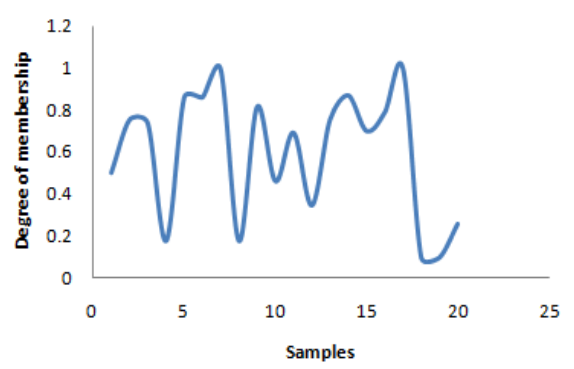

(a) Travelling from Infected Area (TIA)

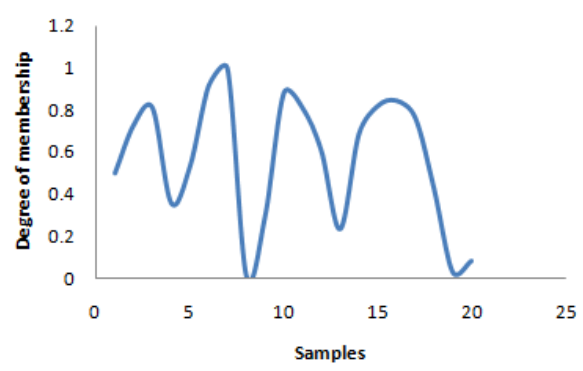

(d) Dry cough (DC)

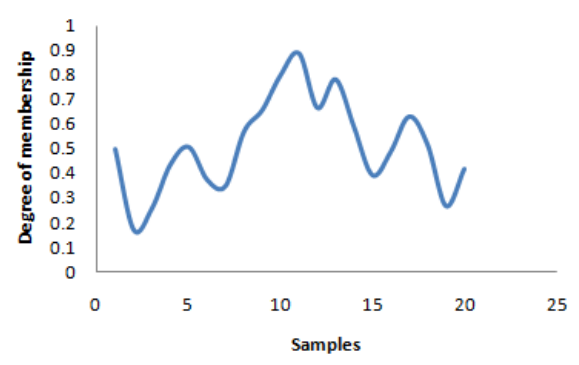

(g) Diarrhea

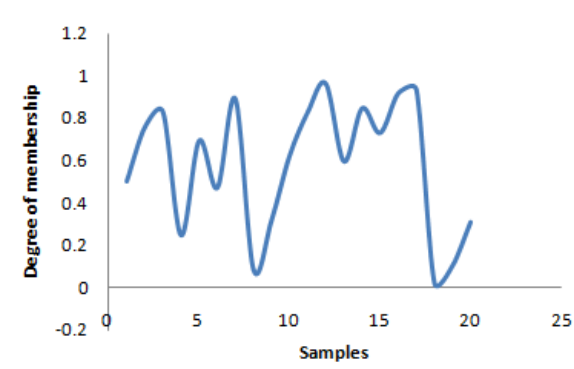

(b) Contact with Infected Person (CIP)

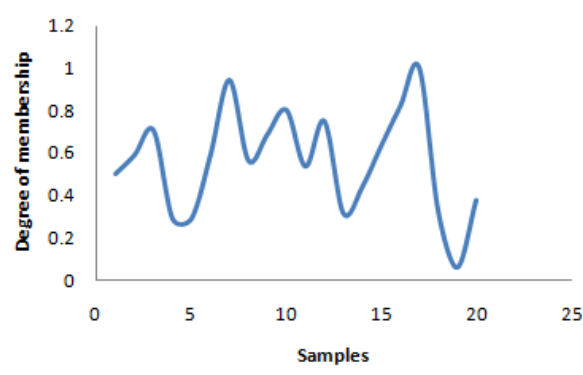

(e) Trouble Breathing (TB)

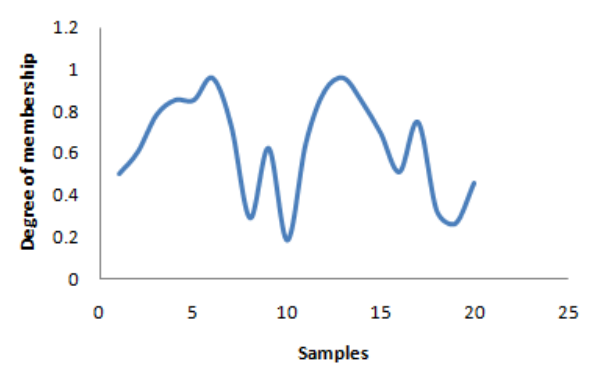

(h) Sore Throat (ST)

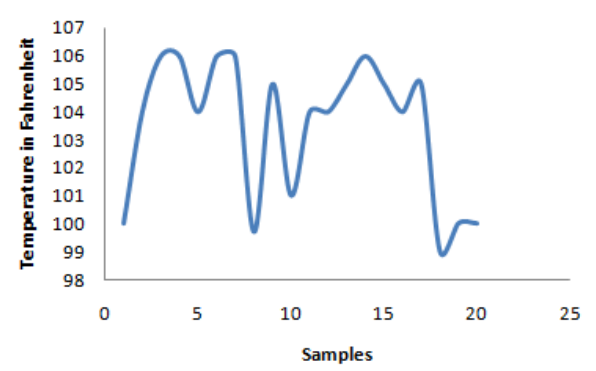

(c) Body Temperature (BT)

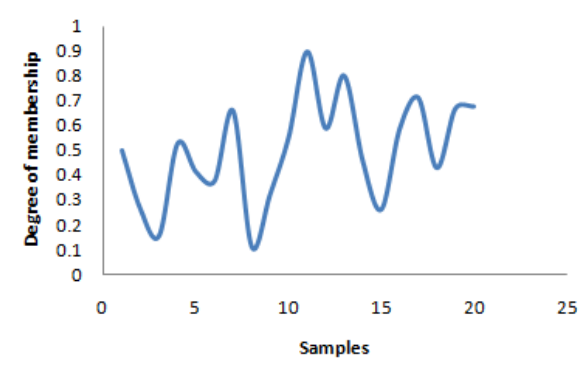

(f) Chest Pain (CP)

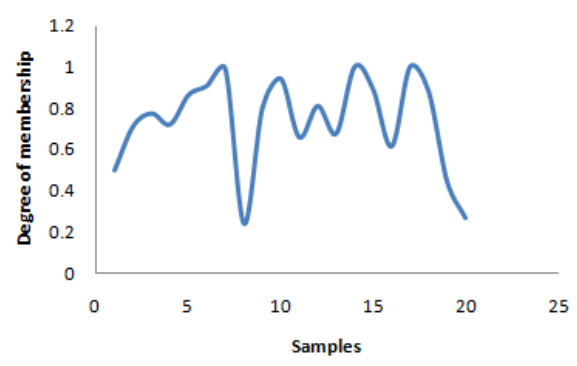

(i) Headache

Fig. 4 Different COVID-19 Symptoms provided from fuzzy logic system as training sets (a-i)

After that, all the 20 patient's data sets and their corresponding symptoms level were expressed in terms of the degree of membership illustrated in Fig. 4, and different outputs representing high, medium, and low infection probability were recorded. These output levels were separated by the crisp value of +1 (red zone), 0 (yellow zone), and -1 (green zone) as shown in Fig. 5 and considered as target data to be trained in neural networks.

\subsection{Design a neural network}

A neural network consists of a processing element with synaptic input connections and a single output. Usually, the inputs $x_{1}, x_{2} \ldots x_{3}$ are multiplied by their respective numeric weights $w_{1}, w_{2} \ldots w_{n}$ and the weighted sum of the input signals can be expressed as [9]:

$n e t=\left(w_{1} \cdot x_{1}+w_{2} \cdot x_{2}+w_{3} \cdot x_{3}+\ldots . .+w_{n} \cdot x_{n}\right)=$ $\left(\sum_{i=1}^{n} w_{i} \cdot x_{i}+b\right)$

Here, $b$ is the threshold value called bias which will always have the value of 1 . The amplitude of the output of a neuron can be limited by a non-linear activation function, $f($.$) as depicted$ in Fig. 6 [9].

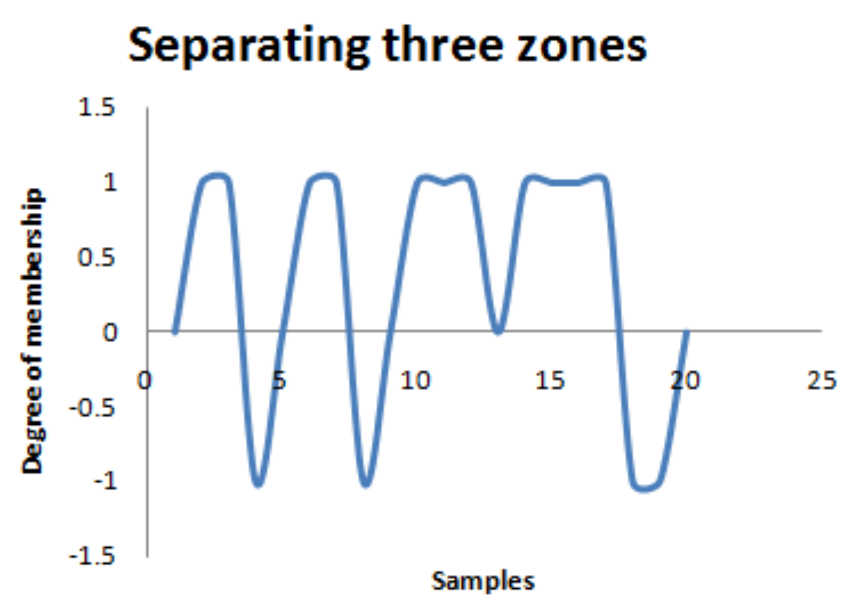

Fig. 5 Target samples or data

In a layered neural network, the neurons are organized in the form of layers. In the simplest form of a layered network, an input layer of source nodes is directly projected onto an output layer of neurons. 


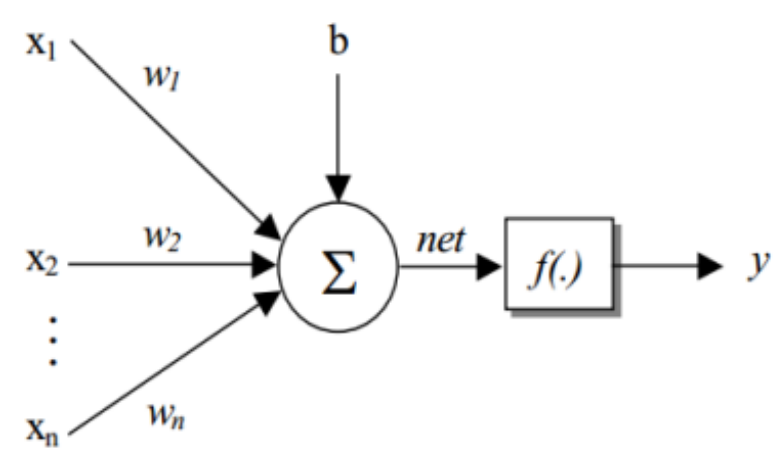

Fig. 6 Perceptron Model

This type of network is known as feedforward as only the forward connectivity of the neurons is considered [10]. Fig. 7 shows such a feedforward network where "single-layer" referring to the output layer of computation nodes (neurons) [9].

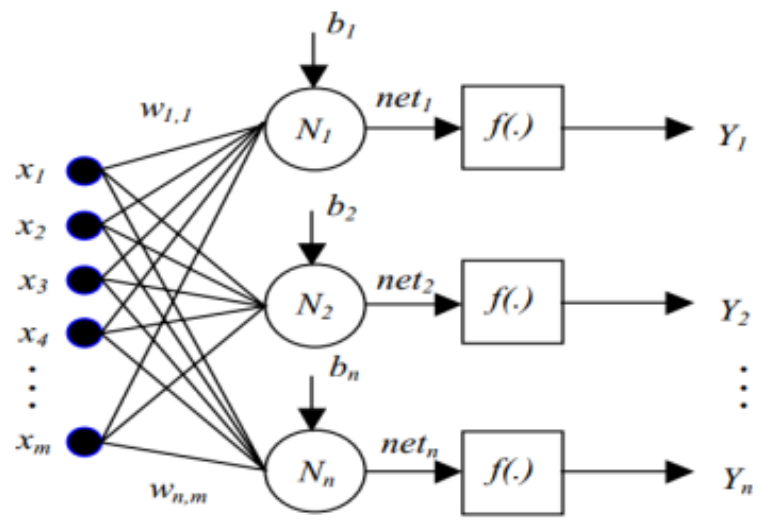

Fig. 7 Single layer feedforward network

Table 1 Rule insertion for zone separation

\begin{tabular}{|c|c|c|c|c|}
\hline \multicolumn{5}{|c|}{ IF } \\
\hline TIA & No & No & Yes & No \\
\hline CIP & No & No & Yes & Yes \\
\hline BT & Low & High & Medium & High \\
\hline$\overline{D C}$ & No & Yes & Yes & Yes \\
\hline TB & No & No & No & Yes \\
\hline $\mathbf{C P}$ & No & No & No & Yes \\
\hline Diarrhea & Sometimes & Sometimes & Sometimes & Sometimes \\
\hline ST & No & Yes & No & Yes \\
\hline Headache & Yes & Yes & Yes & Yes \\
\hline \multicolumn{5}{|c|}{ THEN } \\
\hline $\begin{array}{l}\text { Target } \\
\text { output }\end{array}$ & $\begin{array}{c}-1 \\
\text { (Green } \\
\text { Zone) }\end{array}$ & $\begin{array}{c}0 \\
\text { (Yellow } \\
\text { Zone) }\end{array}$ & $\begin{array}{c}1 \\
\text { (Red } \\
\text { Zone) }\end{array}$ & $\begin{array}{c}1 \\
\text { (Red } \\
\text { Zone) }\end{array}$ \\
\hline
\end{tabular}

$* \mathrm{TIA}=$ Recent travel from infected area, $\mathrm{CIP}=$ Close contact with infected person, $\mathrm{BT}=$ Body temperature, $\mathrm{DC}=$ Dry cough, $\mathrm{TB}=$ Trouble breathing, $\mathrm{CP}=$ Chest pain, $\mathrm{ST}=$ Sore throat.

The inputs of this network are the input vectors consisting different types of COVID-19 symptoms, weights of the network are the weight matrices, and biases are the bias vectors. The target data is output vectors consisting three different values -1 , 0 and 1 representing green zone, yellow zone and red zone, respectively. Three networks such as zone_network1, zone_network2, and zone_network3 were generated with 10, 12 and 14 number of neurons respectively using the backpropagation algorithm as shown in Fig. 8. At first, the nine (09) major symptoms of COVID-19 was converted into linguistic variables and then, different IF----THEN rules were implemented in Mamdani fuzzy inference system listed in Table 1.

Thus, for different combination of major COVID-19 symptoms, different decisions were identified by experts.

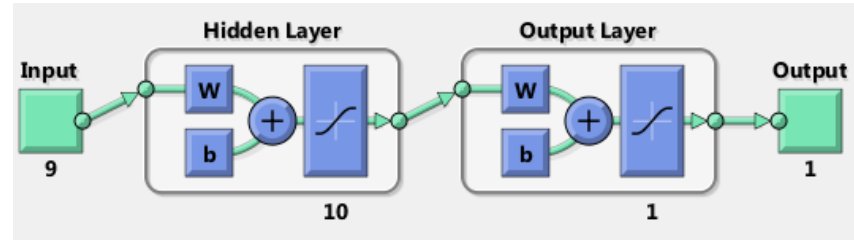

(a) zone_network1

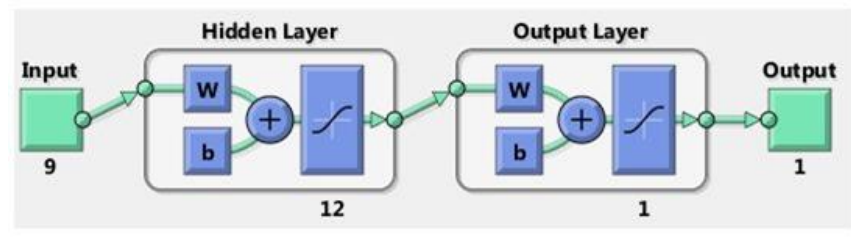

(b) zone_network2

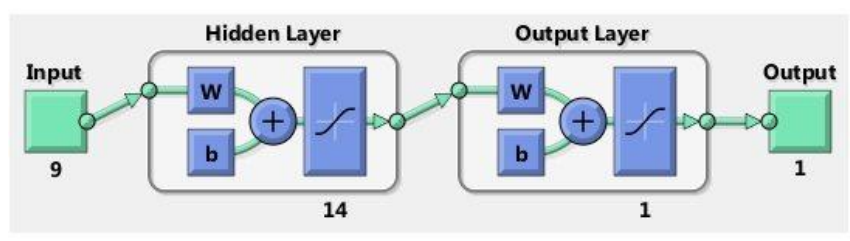

(c) zone_network3

Fig. 8 Network named zone network with 10, 12 and 14 neurons obtained during training process

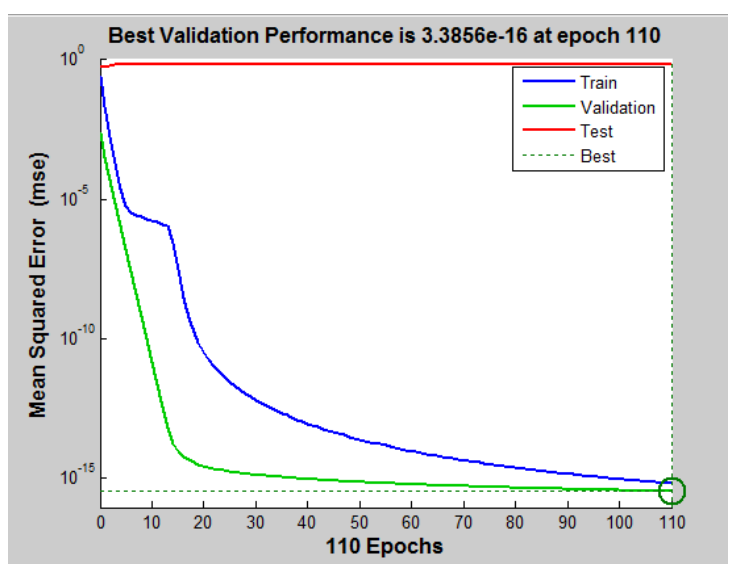

Fig. 9 Performance evaluation of training, validation and test sets in MATLAB

\subsection{Train the neural network for the target data}

The back-propagation algorithm trains the neural network by adjusting its weights [11]. The training process continues till the neural network continues to improve on the validation set. Each network accuracy was measured by test set and the performance was evaluated in terms of logarithmic mean squared error as depicted in Fig. 9. It is evident from Fig. 9 that the mean square error reduces quickly as the number of epoch's increases. 
The algorithm and process information of this training process are listed in Table 2.

Table 2 Algorithm and process information of training

\begin{tabular}{|c|c|}
\hline \multicolumn{2}{|c|}{ Algorithm and process information } \\
\hline Data Division & Random \\
\hline Training & Levenberg-Marquardt \\
\hline Performance & Mean Squared Error (MSE) \\
\hline Epoch & 110 iterations \\
\hline Time & $0: 00: 04$ \\
\hline
\end{tabular}

\subsection{Test the Network}

In this step, the trained neural network was tested with realworld COVD-19 symptoms data set [12]. This testing estimates how well the neural network performs with the actual data set. The tested network generated outputs in the range of -1 to +1 as shown in Fig. 10. The value of nearly or exactly -1 indicates the state of low or no infected area whereas 0 defines the state of medium level of the infected area and +1 defines the state of the high infected area.

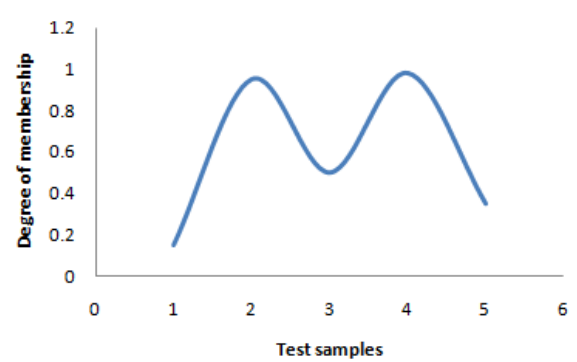

(a) Travelling from Infected Area (TIA)

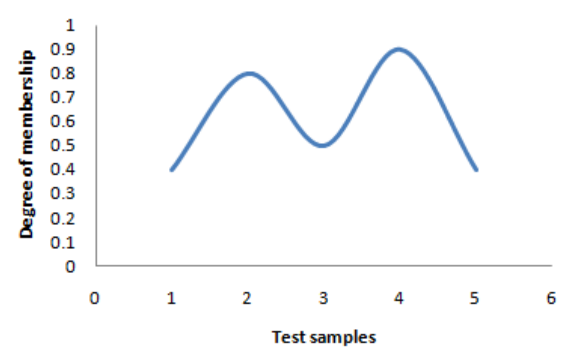

(d) Dry cough (DC)

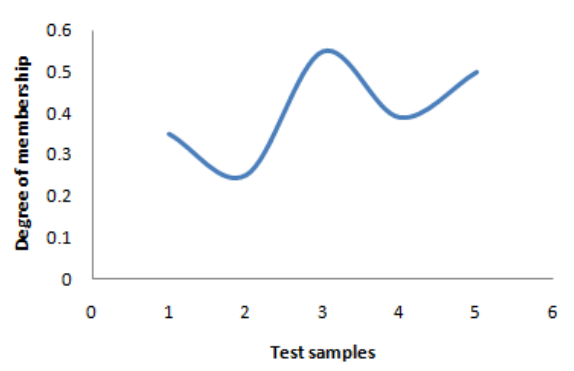

(g) Diarrhea

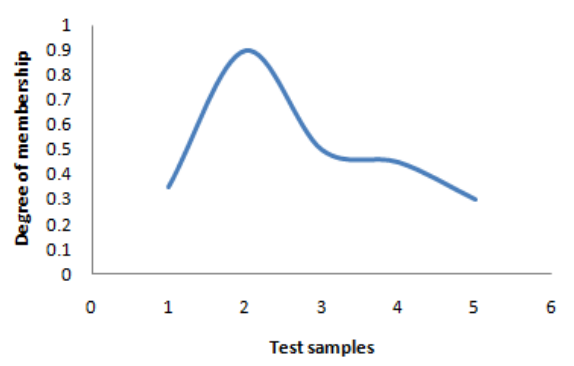

(b) Contact with Infected Person (CIP)

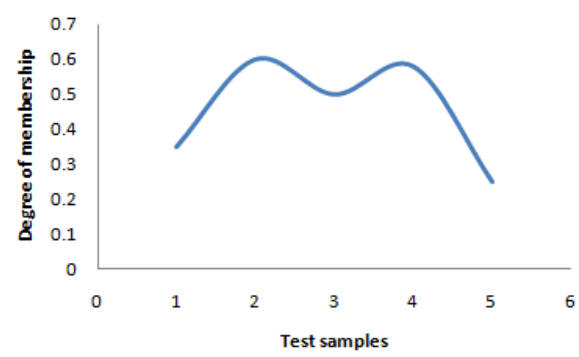

(e) Trouble Breathing (TB)

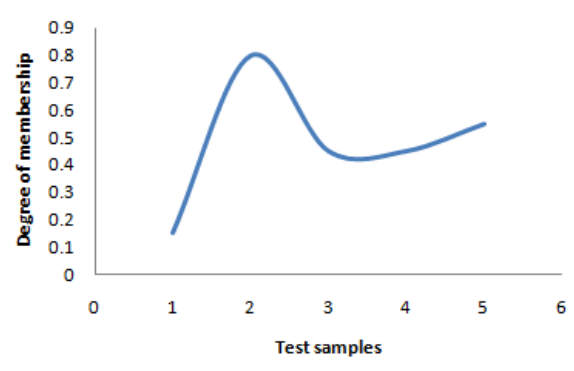

(h) Sore Throat (ST)

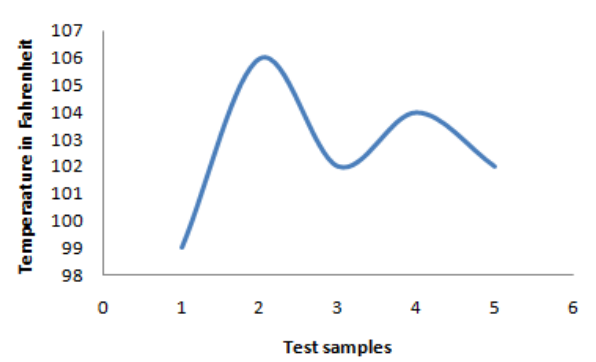

(c) Body Temperature (BT)

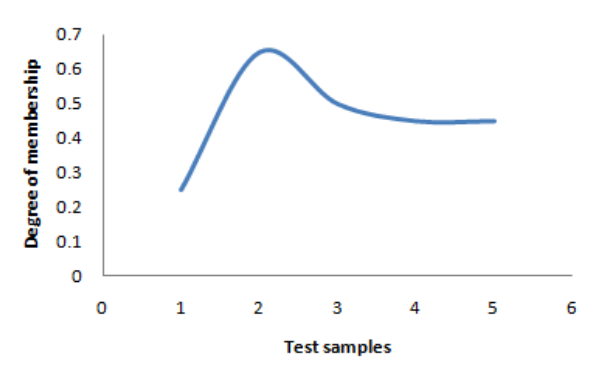

(f) Chest Pain (CP)

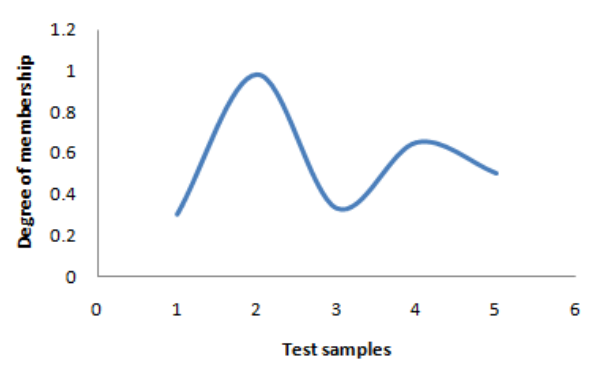

(i) Headache

Fig. 10 Different COVID-19 Symptoms provided from real world as testing sets (a-i)

\section{Results and Discussion}

In this work, different combinations of COVID-19 symptoms were used as linguistic variables of the fuzzy logic system and outputs provided by the experts considered as the target or desired outputs. The neural network was trained with these target outputs. If the network is satisfactorily trained, it is expected that any combinations of the test data or samples would like to follow the desired or target output. To validate this assumption, the neural network was tested by five (05) different combinations of COVID-19 symptoms provided from real-world patients and the predicted outputs of zone_network1, zone_network2, and zone_network3 were listed in Table 3-Table 5 respectively.
It can be seen from Table 3-Table 5 that the predicted output gets closer to the target output with the number of neurons. For instance, person-1 having COVID-19 symptoms resulted in a predicted output of -0.89 with 10 neurons, -0.92 with 12 neurons, and -0.96 with 14 neurons in zone_network1, zone_network2, and zone_network3, respectively.

This dependency on neurons is extensively studied in the Fig. 11 where it is obvious that the chances of getting authoritative desired output increases with the number of neurons at the cost of complexity. 
Table 3 Predicted output with zone_network1

\begin{tabular}{|c|c|c|c|c|c|}
\hline Symptoms & Person-1 & Person-2 & Person-3 & Person-4 & Person-5 \\
\hline TIA & 0.25 & 0.45 & 0.6 & 0.15 & 0.9 \\
\hline CIP & 0.34 & 0.35 & 0.6 & 0.14 & 0.9 \\
\hline BT & 0.45 & 0.5 & 0.5 & 0.25 & 0.85 \\
\hline DC & 0.23 & 0.45 & 0.55 & 0.23 & 0.75 \\
\hline TB & 0.16 & 0.35 & 0.45 & 0.16 & 0.8 \\
\hline CP & 0.1 & 0.1 & 0.45 & 0.15 & 0.75 \\
\hline Diarrhea & 0.25 & 0.35 & 0.75 & 0.35 & 0.65 \\
\hline ST & 0.2 & 0.45 & 0.5 & 0.4 & 0.8 \\
\hline Headache & 0.1 & 0.45 & 0.35 & 0.4 & 0.8 \\
\hline $\begin{array}{c}\text { Target } \\
\text { output }\end{array}$ & -1 & 0 & 1 & -1 & 1 \\
\hline $\begin{array}{c}\text { Predicted } \\
\text { output }\end{array}$ & -0.89 & 0.14 & 0.88 & -0.86 & 0.93 \\
\hline $\begin{array}{c}\text { Zone } \\
\text { Green }\end{array}$ & Yellow & Red & Green & Red \\
\hline
\end{tabular}

Table 4 Predicted output with zone_network2

\begin{tabular}{|c|c|c|c|c|c|}
\hline Symptoms & Person-1 & Person-2 & Person-3 & Person-4 & Person-5 \\
\hline TIA & 0.25 & 0.45 & 0.6 & 0.15 & 0.9 \\
\hline CIP & 0.34 & 0.35 & 0.6 & 0.14 & 0.9 \\
\hline BT & 0.45 & 0.5 & 0.5 & 0.25 & 0.85 \\
\hline DC & 0.23 & 0.45 & 0.55 & 0.23 & 0.75 \\
\hline TB & 0.16 & 0.35 & 0.45 & 0.16 & 0.8 \\
\hline CP & 0.1 & 0.1 & 0.45 & 0.15 & 0.75 \\
\hline Diarrhea & 0.25 & 0.35 & 0.75 & 0.35 & 0.65 \\
\hline ST & 0.2 & 0.45 & 0.5 & 0.4 & 0.8 \\
\hline Headache & 0.1 & 0.45 & 0.35 & 0.4 & 0.8 \\
\hline $\begin{array}{c}\text { Target } \\
\text { output }\end{array}$ & -1 & 0 & 1 & -1 & 1 \\
\hline $\begin{array}{c}\text { Predicted } \\
\text { output }\end{array}$ & -0.92 & 0.08 & 0.91 & -0.94 & 0.95 \\
\hline $\begin{array}{c}\text { Zone } \\
\text { Green }\end{array}$ & Yellow & Red & Green & Red \\
\hline
\end{tabular}

Table 5 Predicted output with zone_network3

\begin{tabular}{|c|c|c|c|c|c|}
\hline Symptoms & Person-1 & Person-2 & Person-3 & Person-4 & Person-5 \\
\hline TIA & 0.25 & 0.45 & 0.6 & 0.15 & 0.9 \\
\hline CIP & 0.34 & 0.35 & 0.6 & 0.14 & 0.9 \\
\hline BT & 0.45 & 0.5 & 0.5 & 0.25 & 0.85 \\
\hline DC & 0.23 & 0.45 & 0.55 & 0.23 & 0.75 \\
\hline TB & 0.16 & 0.35 & 0.45 & 0.16 & 0.8 \\
\hline CP & 0.1 & 0.1 & 0.45 & 0.15 & 0.75 \\
\hline Diarrhea & 0.25 & 0.35 & 0.75 & 0.35 & 0.65 \\
\hline ST & 0.2 & 0.45 & 0.5 & 0.4 & 0.8 \\
\hline Headache & 0.1 & 0.45 & 0.35 & 0.4 & 0.8 \\
\hline $\begin{array}{c}\text { Target } \\
\text { output }\end{array}$ & -1 & 0 & 1 & -1 & 1 \\
\hline $\begin{array}{c}\text { Predicted } \\
\text { output }\end{array}$ & -0.96 & 0.04 & 0.97 & -0.98 & 0.98 \\
\hline $\begin{array}{c}\text { Zone } \\
\text { Green }\end{array}$ & Yellow & Red & Green & Red \\
\hline
\end{tabular}

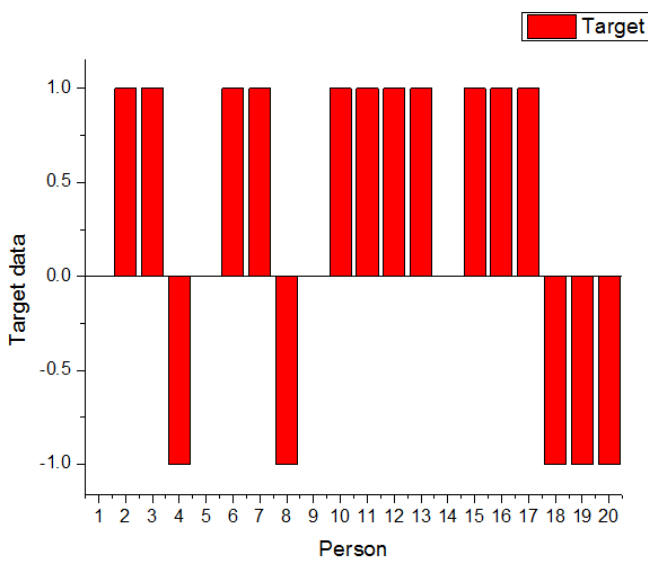

(a) Target data

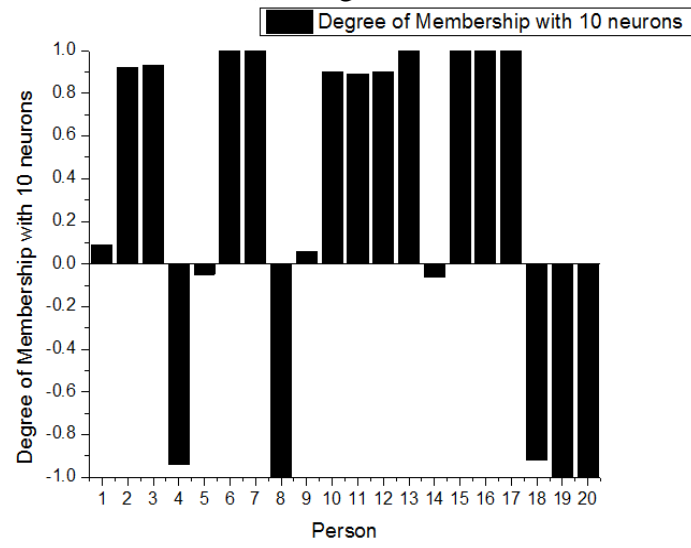

(b) 10 neurons

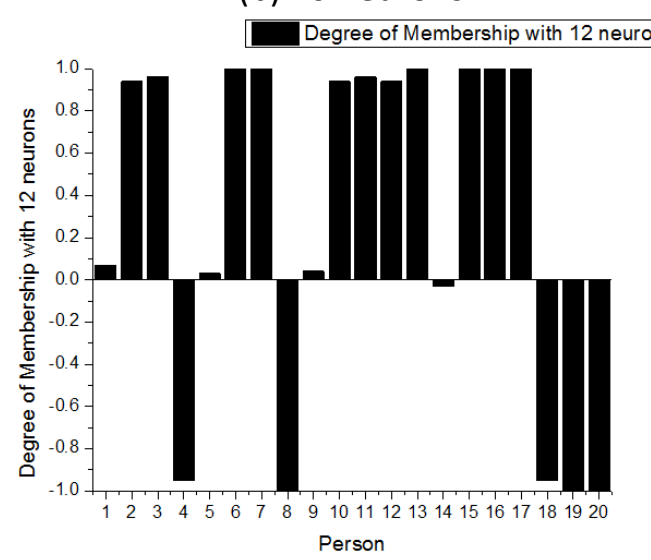

(c) 12 neurons

Degree of Membership with 14 neurons

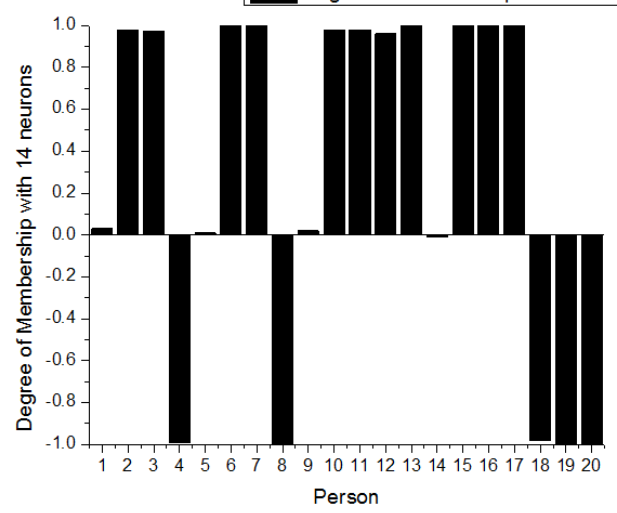

(d) 14 neurons

Fig. 11 Performance evaluation of actual and multilayer protocol output of COVID-19 symptoms 
Thus, the fuzzy logic output was trained into the neural network in such a way that the threshold value of separating country into three zones: green, yellow, and red can be adapted according to miscellaneous symptoms situations of COVID-19.

\section{Conclusion}

For the last several months, the COVID-19 outbreak quickly surges worldwide. To control the COVID-19 infection cases government of many countries planned to imply zonal lockdown: red, yellow, and green in its territory based on the number of COVID-19 confirmed cases. Due to the lack of COVID-19 testing kit availability, social awareness, cost, or other reasons, the exact number of COVID-19 positive cases cannot be traced yet. Therefore, it would not be wise to divide such zones based on the infected number only. In this paper, we proposed an artificial neural network generated logical decisions based on COVID-19 symptoms by which a country or region can be divided into red, yellow, and green zone representing the highly infected areas, moderate-infected area, and controlled or low infected area, respectively. To train the neural network, the data sets were generated from the different combinations of COVID19 symptoms. At first, an online survey of twenty (20) patients was conducted based on the nine (09) major symptoms of COVID-19. This survey information was implemented in the Mamdani inference system where these 09 symptoms were introduced as linguistic variables and their corresponding possible output recommendations were recorded. Then, different fuzzy rules were inserted in the Mamdani inference system and evaluated for possible recommendations. Based on these rules, three different outputs were generated representing highly infected area (red zone), moderately infected area (yellow zone), and controlled or low infected area (green zone), respectively. These outputs were considered as target data to be trained in neural networks. After the training, the neural network was tested by five (05) different combinations of COVID-19 symptoms provided from real-world patients. The accuracy of the predicted output gets closer to the target output with the number of neurons in the hidden layer.

\section{References}

[1] https://www.worldometers.info/coronavirus/?fbclid=IwA R2kywwEIfUIKzsE7bkK1KOHna_MR66fHZS0E_RAjrf hOtJchGxIxUgrf2o
[2] Dhiman, N. and Sharma, M.K., 2020. Fuzzy Logic Inference System for Identification and Prevention of Coronavirus (COVID-19). International Journal of Innovative Technology and Exploring Engineering (IJITEE), 9(6), pp.1575-1580.

[3] https://www.who.int/docs/defaultsource/coronaviruse/situation-reports/20200423-sitrep94-covid19.pdf?sfvrsn=b8304bf0_2\#: :text=Retrospective $\% 20$ investigations $\% 20$ by $\% 20$ Chinese $\% 20$ authorities, $\% 2$ C\%20some\%20did\%20not, last accessed 2020/12/16.

[4] https://www.who.int/docs/defaultsource/coronaviruse/situation-reports/20200225-sitrep36-covid19.pdf?sfvrsn=2791b4e0_2\#: :text=Although\%20for $\% 2$ 0most\%20people\%20COVID,to\%20be\%20more\%20vul nerable, last accessed 2020/12/16.

[5] Centers for Disease Control and Prevention | HealthData.gov, https://healthdata.gov/agencies/centersdisease-control-and-prevention, last accessed 2020/12/16.

[6] Tamang, S.K., Singh, P.D. and Datta, B., 2020. Forecasting of Covid-19 cases based on prediction using artificial neural network curve fitting technique. Global Journal of Environmental Science and Management, 6(Special Issue (Covid-19)), pp.53-64.

[7] Coronavirus (COVID-19) Overview, https://www.webmd.com/lung/coronavirus, last accessed 2020/12/16.

[8] Larsen, J.R., Martin, M.R., Martin, J.D., Kuhn, P. and Hicks, J.B., 2020. Modeling the Onset of Symptoms of COVID-19. Frontiers in public health, 8, p.473.

[9] Siddique, N. and Adeli, H., 2013. Computational intelligence: synergies of fuzzy logic, neural networks and evolutionary computing. John Wiley \& Sons.

[10] Haykin, S., 2009. Neural Networks and Learning Machines, Prentice Hall, New York

[11] Hecht-Nielsen, R., 1992. Theory of the backpropagation neural network. In Neural networks for perception (pp. 65-93). Academic Press.

[12] Coronavirus Symptoms (COVID-19) - Worldometer, https://www.worldometers.info/coronavirus/coronavirussymptoms/, last accessed 2020/12/18. 